\title{
Die Schweizerische Stiftung für Gesundheitserziehung (SSGE)
}

Barbara Zumstein

Sumatrastrasse 30, 8006 Zürich

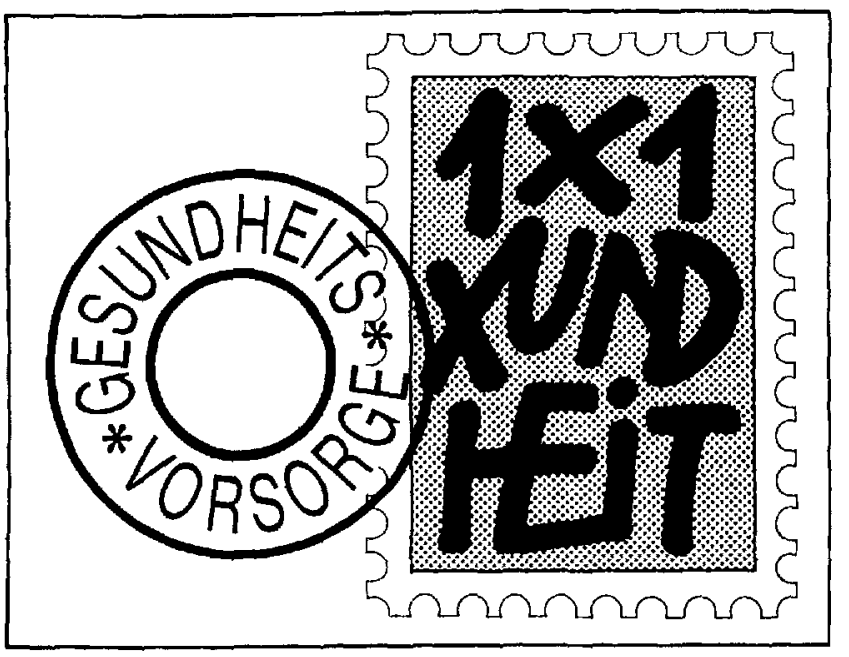

Es ist der Initiative der Schweizerischen Gesellschaft für Sozial- und Präventivmedizin zu verdanken, dass Ende 1972 die SSGE mit einem Startkapital von seiten der Bundesfeierspende gegründet werden konnte.

Die SSGE beschäftigt sich landesweit mit primärer Prophylaxe; der Förderung der gesundheitlichen Erziehung, Dokumentation und Information von Kindern, Jugendlichen, Ärzten, Schulen, Behörden und interessierten Einzelpersonen.

Gesundheitsförderung - gemeindenahe Prävention kommt ohne die Hilfe der Dienste der professionellen Gesundheitserziehung nicht aus. Die SSGE bietet denn auch folgende Dienstleistungen an:

- Beratung von natürlichen Personen, Behörden und Schulen

- Erstellen von Dokumentationen $\mathrm{zu}$ bestimmten aktuellen Fragen

- Mitarbeit bei Projekten im Gesundheitsbereich

- Erstellen von Medienpaketen für die Schulen

- Erarbeiten von Broschüren

- Durchführung von Seminarien

- Vermittlung von Kontakten

- Mitarbeit bei Ausstellungen, Erhebungen und firmeneigenen Kampagnen

In Lugano hat sich die SSGE denn auch mit einem eigenen Ausstellungsstand beteiligt. Vorgestellt wurden die Materialien, welche die SSGE den in der Gesundheitsförderung Beschäftigen vor Ort anbieten kann:

\section{Medienpaket «1 $1 \times 1$ Xundheit *}

In den Gesundheitskrimis, die von Radio DRS mehrmals ausgestrahlt wurden, leistet Xandi Merk Aufklä- rungsarbeit in den Fällen Herzinfarkt, chronische Bronchitis, Übergewicht, Alkoholleber, Verstopfung, Kopfweh, Karies, Grippe, Rückenleiden und Nierensteine. Das Medienpaket umfasst Hörspiele, eine Begleitbroschüre, zehn Merkblätter, zehn Poster und zwölf Hellraumprojektorfolien.

\section{«Unterrichtseinheit Grundkenntnisse einer gesunden Ernährung»}

Die dreiteilige Unterrichtseinheit ist im Baukastensystem aufgebaut und richtet sich an Schüler im Alter von 12-16 Jahren und ihre Lehrer.

Daneben werden z.Z. Manuals für die Ernährungserziehung im Kindergarten, für übergewichtige Kinder, für ernährungsbewusste Personalverpflegung, eine Projektwoche zum Thema Tabakmissbrauch und für eine Aktionswoche Unfallverhütung in der Gemeinde erstellt.

\section{Summary}

\section{Swiss Foundation of Health Education}

The Swiss Foundation of Health Education has been founded in 1972. Its functions are: primary prophylacty, promotion of health education, documentation and public information. The Foundation

- gives advices in questions of health for all persons interested

- maintains a documentation of actual problems

- co-operates in different projects with other institutions

- develops documents for use in schools

- creates booklets and courses

- organizes meetings.

\section{Résumé}

Fondation sujsse pour l'éducation à la santé

C'est grâce à l'initiative de la Société suisse de médecine sociale et préventive et à l'aide d'une contribution financiaire du Don suisse de la Fête nationale que la Fondation suisse pour l'éducation à la santé a pu être fondée en 1972. La fondation favorise la prévention primaire et l'éducation à la santé avec l'assistance d'un centre de documentation et offre les services suivants:

- Conseil de toutes les personnes, autorités et écoles intéressées par les problèmes de la santé

- Documentation sur des questions actuelles

- Coopération à plusieurs projets de santé

- Développement de documents audio-visuels pour les écoles

- Création de brochures

- Réalisation de cours

- Etablissement de contacts avec des tiers

- Collaboration à des expositions et sondages

- Développement de projets internes. 